\title{
(-)-Epigallocatechin-3-gallate induces apoptosis in human pancreatic cancer cells via PTEN
}

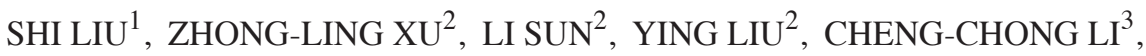 \\ HONG-MEI LI ${ }^{3}$, WEI ZHANG ${ }^{2}$, CHENG-JUN LI ${ }^{3}$ and WEI QIN ${ }^{3}$ \\ Departments of ${ }^{1}$ General Surgery and ${ }^{2}$ Oncology, The Third Affiliated Hospital of Qiqihar Medical University; \\ ${ }^{3}$ Department of Pharmaceutical Science, Qiqihar Medical University, Qiqihar, Heilongjiang 161099, P.R. China
}

Received May 28, 2015; Accepted April 6, 2016

DOI: $10.3892 / \mathrm{mmr} .2016 .5277$

\begin{abstract}
Phosphatase and tensin homolog deleted on chromosome 10 (PTEN) is a cancer suppressor gene and an important negative regulator in the phosphatidylinositide 3-kinase (PI3K)/protein kinase B (Akt)/ mechanistic target of rapamycin (mTOR) signaling pathway. The PI3K/Akt/mTOR pathway can promote cancer cell survival, proliferation and progression. In the present study, the effects of (-)-epigallocatechin-3-gallate (EGCG) on PI3K/Akt/mTOR signaling in pancreatic cancer cells and PTEN knockdown cells were measured, in addition to assessing its therapeutic potential in pancreatic cancer. The apoptosis and proliferation of the cancer cells were examined by flow cytometry and Cell Counting kit- 8 assay, respectively. The expression of genes and proteins in the PI3K/Akt/mTOR signaling pathway were investigated by reverse transcription-polymerase chain reaction and western blotting, respectively. The results suggested that the EGCG-induced apoptosis, proliferation inhibition and downregulated expression of phosphorylated (p)-Akt and p-mTOR were partially attenuated in PTEN-knockdown cells. In conclusion, the results indicated that EGCG is able to reduce proliferation and induce the apoptosis of pancreatic cancer cells associated with the expression of PTEN. Additionally, EGCG can suppress the expression of p-Akt and p-mTOR via PTEN to regulate the PI3K/Akt/mTOR pathway. The results suggest that EGCG may represent a potential treatment for pancreatic cancer, based on PTEN activation.
\end{abstract}

Correspondence to: Dr Shi Liu, Department of General Surgery, The Third Affiliated Hospital of Qiqihar Medical University, 27 Taishun Road, Qiqihar, Heilongjiang 161099, P.R. China E-mail: shiliu2199@163.com

Key words: pancreatic cancer, (-)-epigallocatechin-3-gallate, phosphatase and tensin homolog deleted on the chromosome ten, phosphatidylinositol3-kinase, protein kinase B, mechanistic target of rapamycin

\section{Introduction}

Pancreatic cancer (PC) is one of the most malignant tumors in humans, and its 5-year survival rate is $<5 \%$ (1). Additionally, the development and metastasis of PC frequently goes undetected, and in China $\sim 80 \%$ of patients with PC are inoperable (2). Unfortunately, the incidence of PC is increasing, therefore, effective new medicines and therapeutic targets are required.

Green tea is a popular drink consumed worldwide, and a number of epidemiological studies have indicated an association between tea consumption and reduced incidence of cancer (3-5). (-)-Epigallocatechin-3-gallate (EGCG) is the most abundant catechin in green tea, and has been shown to inhibit inflammation, oxidation, viruses and oncogenesis (6-9). EGCG acts on numerous molecular targets, and has been demonstrated to have inhibitory ability during the initiation and progression stages of carcinogenesis (10-12). It can inhibit the growth and metastasis of a number of types of cancer (13-15) through a variety of mechanisms $(16,17)$, including modulation of the phosphatidylinositide 3-kinase $(\mathrm{PI} 3 \mathrm{~K}) /$ protein kinase $\mathrm{B}(\mathrm{Akt}) /$ mechanistic target of rapamycin (mTOR) pathway (18).

PTEN is a potent tumor-suppressor gene and a significant negative regulator of the PI3K/Akt/mTOR pathway. The PI3K/Akt/mTOR pathway modulates cellular functions, including proliferation, differentiation and migration (19). The dysregulation of this pathway has been associated with many types of cancer (20), including PC. PI3K/Akt/mTOR pathway activity promotes cancer cell proliferation, invasion and metastasis and inhibits apoptosis (21).

In a previous study, EGCG was demonstrated to upregulate the expression of PTEN and downregulate the expression of phosphorylated (p)-Akt and p-mTOR in human PC cells (22). In the present study, PC cells with or without PTEN knockdown were treated with EGCG, and the alterations in apoptosis and protein expression of $\mathrm{PI} 3 \mathrm{~K} / \mathrm{Akt} / \mathrm{mTOR}$ pathway targets were examined to investigate the therapeutic mechanisms of EGCG in human PC.

\section{Materials and methods}

Lentiviral-based RNA interference knockdown of PTEN in $P C$ cells. The human PC cell lines, PANC-1 and BxPC-3, 
were purchased from the Shanghai Institute of Biochemistry and Cell Biology, Chinese Academy of Sciences (Shanghai, China). PTEN knockdown in PANC-1 and BxPC-3 cells was performed as previously described (23). Briefly, lentiviral transduction was used to steadily express short hairpin RNAs (shRNAs) that target PTEN. shRNA constructs were obtained from Sigma-Aldrich (St. Louis, MO, USA). Both the PTEN shRNA construct (TRCN0000219043, including the shRNA for human PTEN) and the luciferase shRNA construct (TRCN0000072247, including the shRNA as a control) were used to produce recombinant lentiviral particles. The PC cells were transfected with the viral particles containing PTEN or luciferase shRNAs for $24 \mathrm{~h}$ using Lipofectamine 2000 (Invitrogen; Thermo Fisher Scientific, Inc., Waltham, MA, USA), after which the cells were placed into fresh RPMI-1640 medium (Gibco; Thermo Fisher Scientific, Inc.). The supernatants were collected at 36, 48, 60 and $72 \mathrm{~h}$ following transduction, and the supernatants were filtered using $0.45 \mu \mathrm{m}$ low protein-binding filters (EMD Millipore, Billerica, MA, USA). Subsequently, the viral particles were centrifuged at $20,000 \times \mathrm{g}$ at $4^{\circ} \mathrm{C}$ for $2 \mathrm{~h}$ and then resuspended in fresh RPMI-1640 medium. The lentiviral particles (shPTEN and shLuc) were then introduced to PANC-1 and BxPC-3 cells at a multiplicity of infection of 40. The PTEN knockdown was examined by Western blotting in triplicate experiments.

Cell culture and treatment. $\mathrm{PC}$ cells were incubated at $37^{\circ} \mathrm{C}$ in a $95 \%$ air and $5 \% \mathrm{CO}_{2}$ atmosphere in Roswell Park Memorial Institute 1640 (Gibco; Thermo Fisher Scientific, Inc.) supplemented with $10 \%$ fetal bovine serum (GE Healthcare Life Sciences, Logan, UT, USA). The cells with or without PTEN knockdown were treated with $40 \mu \mathrm{g} / \mathrm{ml}$ EGCG (Sigma-Aldrich) for $48 \mathrm{~h}$, with control cells treated with deionized water. Subsequently, cell proliferation was examined using a Cell Counting kit-8 (CCK-8) assay. Apoptosis was detected by flow cytometry. The expression of genes and proteins in the $\mathrm{PI} 3 \mathrm{~K} / \mathrm{Akt} / \mathrm{mTOR}$ signaling pathway were analyzed by reverse transcription-polymerase chain reaction (RT-PCR) and western blotting.

Cell proliferation assays. PC cell proliferation was measured by CCK-8 assays as previously described (24). Briefly, the cells (with/without PTEN knockdown) were plated in 96-well plates of 5,000 cells/well. Following culture at $37^{\circ} \mathrm{C}$ for $24 \mathrm{~h}$, the cells were treated with $40 \mu \mathrm{g} / \mathrm{ml}$ EGCG for 24,48 and $72 \mathrm{~h}$. The cells were incubated with CCK-8 (Dojindo Molecular Technologies, Inc., Kumamoto, Japan) solution (10 $\mu \mathrm{l} /$ well) for $2 \mathrm{~h}$, and then the absorbance was measured at $450 \mathrm{~nm}$ using a microplate reader (Model 680; Bio-Rad Laboratories, Inc., Hercules, CA, USA). The alterations in cell growth were calculated as the Inhibition Ratio $(\%)=(1$-treated group OD values/control group OD values) x 100 . The experiments were performed in triplicate.

Apoptosis assays. The apoptosis rates of PANC-1 and BxPC-3 cells were examined using an annexin V-fluorescein isothiocyante (FITC) apoptosis detection kit (BioVision, Inc., Milpitas, CA, USA) as described previously (22). Briefly, the cells were dissociated using trypsin, and $10 \mu \mathrm{l}$ annexin V-FITC and $10 \mu \mathrm{l}$ propidium iodide were then added to the cells in the dark for
$10 \mathrm{~min}$. Stained cells were analysed by flow cytometry using a FACSCalibur flow cytometer (BD Biosciences, Franklin, NJ, USA). Cells in the lower right quadrant of the dot plot were considered to be in early apoptosis, and those in the upper right quadrant were in late apoptosis. The experiments were performed in triplicate.

RT-PCR analysis. PI3K, Akt, PTEN, and mTOR mRNA expression was analyzed by RT-PCR as previously described (22). Glyceraldehyde 3-phosphate dehydrogenase (GAPDH) was used as the internal control. Total RNA was extracted from cells using TRIzol reagent (Invitrogen; Thermo Fisher Scientific, Inc.) and was treated with DNase (Promega Corporation, Madison, WI, USA), prior to reverse transcription into cDNA using the RETROscript ${ }^{\mathrm{TM}}$ kit (cat. no. AM1710; Thermo Fisher Scientific, Inc.), which contained dNTPs, a RNase inhibitor, M-MLV reverse transcriptase and RT buffer (Tris-HCl, $\mathrm{KCl}, \mathrm{MgCl}_{2}$ and DTT). RT-PCR was conducted using an AccessQuick RT-PCR System (Promega Corporation). A total of 30 cycles of amplification were performed using the following conditions: Denaturation at $94^{\circ} \mathrm{C}$ for $30 \mathrm{sec}$, annealing at $58^{\circ} \mathrm{C}$ for $30 \mathrm{sec}$, and extension at $72^{\circ} \mathrm{C}$ for $1 \mathrm{~min}$. The primer sequences were as follows: PI3K forward, 5'-AGGAGCGGTACAGCAAAGAA-3' and reverse, 5'-GCCGAACACCTTTTTGAGTC-3'; AKT forward, 5'-TGA AAACCTTCTGTGGGACC-3' and reverse, 5'-TGGTCCTGG TTGTAGAAGGG-3'; PTEN forward, 5'-CAGAAAGACTTG AAGGCGTAT-3' and reverse, 5'-CGTCGTGTGGGTCCT GAGTGA-3'; mTOR forward, 5'-CTGGGACTCAAATGT GTGCAGTTC-3' and reverse, 5'-GAACAATAGGGTGAA TGATCCGGG-3'; and GAPDH forward, 5'-GGAAGGTGA AGGTCGGAGT-3' and reverse, 5'-CCTGGAAGATGGTGA TGGG-3'. The PCR products were separated by $1 \%$ agarose gel electrophoresis and stained with ethidium bromide, and the results analyzed using NIH Image 1.60 software (National Institutes of Health, Bethesda, MD, USA). The experiments were performed in triplicate.

Western blotting. Protein extraction and western blotting were conducted as previously described (22). In brief, the cells were rinsed with phosphate-buffered saline, and lysed with lysis buffer for $30 \mathrm{~min}$. Subsequently, the lysates were centrifuged at $12,000 \mathrm{xg}$ for $10 \mathrm{~min}$, and the protein concentrations were measured using a bicinchoninic acid protein assay kit (Pierce Biotechnology, Inc., Rockford, IL, USA). Following this, the proteins were separated by $10 \%$ sodium dodecyl sulfate-polyacrylamide gel electrophoresis at $80 \mathrm{~V}$ for $1.5 \mathrm{~h}$, and then transferred onto polyvinylidene fluoride membranes (EMD Millipore) at $100 \mathrm{~V}$ for $2.5 \mathrm{~h}$. Following incubation in bovine serum albumin (Gibco; Thermo Fisher Scientific, Inc.) at $4^{\circ} \mathrm{C}$ for $1 \mathrm{~h}$, the membranes were incubated with the following primary antibodies: Rabbit anti-PTEN (1:1,000; cat. no. 9188), rabbit anti-PI3K (1:1,000; cat. no. 4249), rabbit anti-Akt (1:1,000; cat. no. 4685), mouse anti-p-Akt (1:500; cat. no. 12694), rabbit anti-mTOR (1:1,000; cat. no. 2983), rabbit anti-p-mTOR (1:1,000; cat. no. 5536) and mouse anti- $\beta$-actin (1:500; cat. no. 3700) monoclonal antibodies (Cell Signaling Technology, Inc., Danvers, MA, USA) in Tris-buffered saline-Tween-20; (Sigma-Aldrich) overnight. Membranes were then incubated with horseradish peroxidase-conjugated 

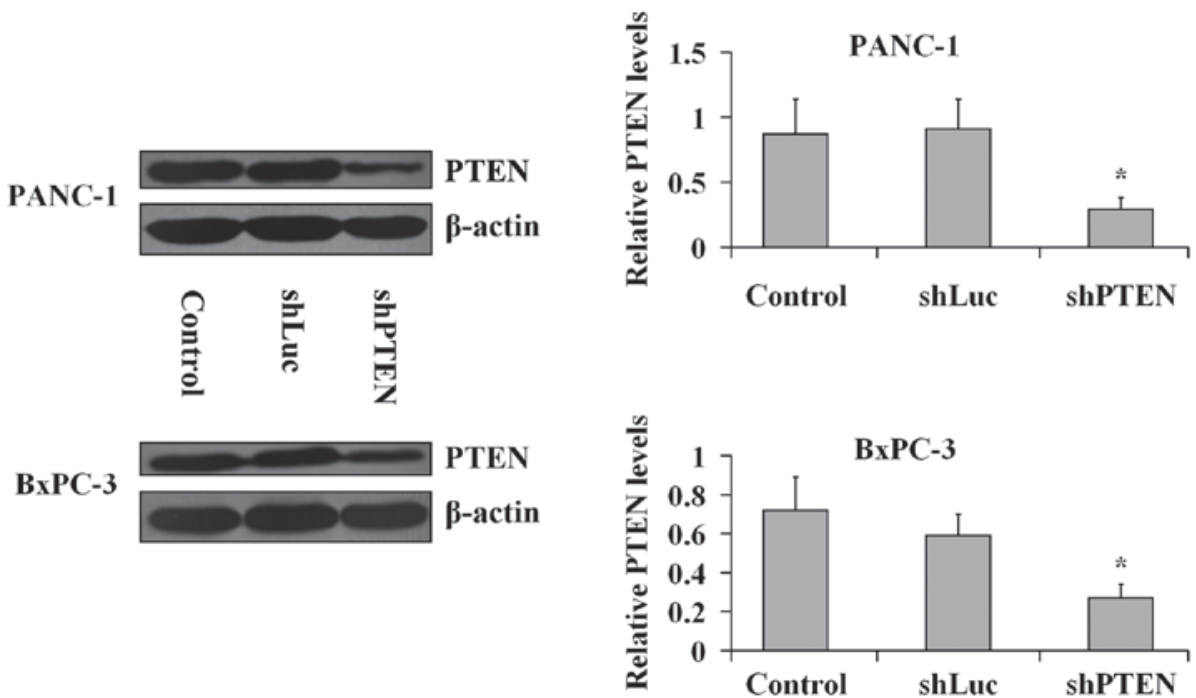

Figure 1. PTEN knockdown by RNA interference in prostate cancer cells. PANC-1 and BxPC-3 cells were transfected with viral particles containing PTEN or Luc shRNAs for $24 \mathrm{~h}$ and the knockdown of PTEN confirmed by western blotting analysis. " $\mathrm{P}<0.05$ vs. control group (untreated cells). PTEN, phosphatase and tensin homolog deleted on chromosome 10; Luc, luciferase; shRNA, short hairpin RNA.

goat anti-rabbit (1:1,000; cat. no. 7074) and goat anti-mouse (1:2,500; cat. no. 7076) secondary antibodies (Cell Signaling Technology, Inc.). Following rinsing, the bands were detected using an enhanced chemiluminescence detection system (GE Healthcare Life Sciences, Chalfont, UK). Relative protein levels were normalized to $\beta$-actin as the internal control. The experiments were performed in triplicate.

Statistical analysis. Statistical analysis was performed using SPSS software, version 13.0 (SPSS, Inc., Chicago, IL, USA). The data are presented as the mean \pm standard deviation. Differences between groups were examined using one-way analysis of variance followed by Fisher's least significant difference test. $\mathrm{P}<0.05$ was considered to indicate a statistically significant difference.

\section{Results}

PTEN knockdown by RNA interference in PC cells. PANC-1 and $\mathrm{BxPC}-3$ cells were transfected with the viral particles including PTEN or luciferase shRNAs for $24 \mathrm{~h}$ and the knockdown of PTEN was confirmed by western blotting analysis (Fig. 1). The $\beta$-actin expression levels in the treated group did not differ with the levels in the control (untreated) group $(\mathrm{P}>0.05)$. PTEN expression levels were significantly lower in shPTEN groups compared with the control group $(\mathrm{P}<0.05)$, Furthermore, the expression levels of PTEN in the shLuc groups were not different compared with the controls $(\mathrm{P}>0.05)$.

EGCG inhibits PC cell proliferation via PTEN. PANC-1 and BxPC-3 cells with or without PTEN knockdown were cultured in medium with or without $40 \mu \mathrm{g} / \mathrm{ml} \mathrm{EGCG} \mathrm{for} 24$, 48 and $72 \mathrm{~h}$, and proliferation was examined by CCK-8 assays (Fig. 2). In PANC-1 cells, the inhibition ratio in the EGCG group at 48 and $72 \mathrm{~h}$ was significantly higher compared with the shPTEN group $(\mathrm{P}<0.05$ and $\mathrm{P}<0.01$, respectively). Furthermore, the inhibition ratio in the shPTEN+EGCG
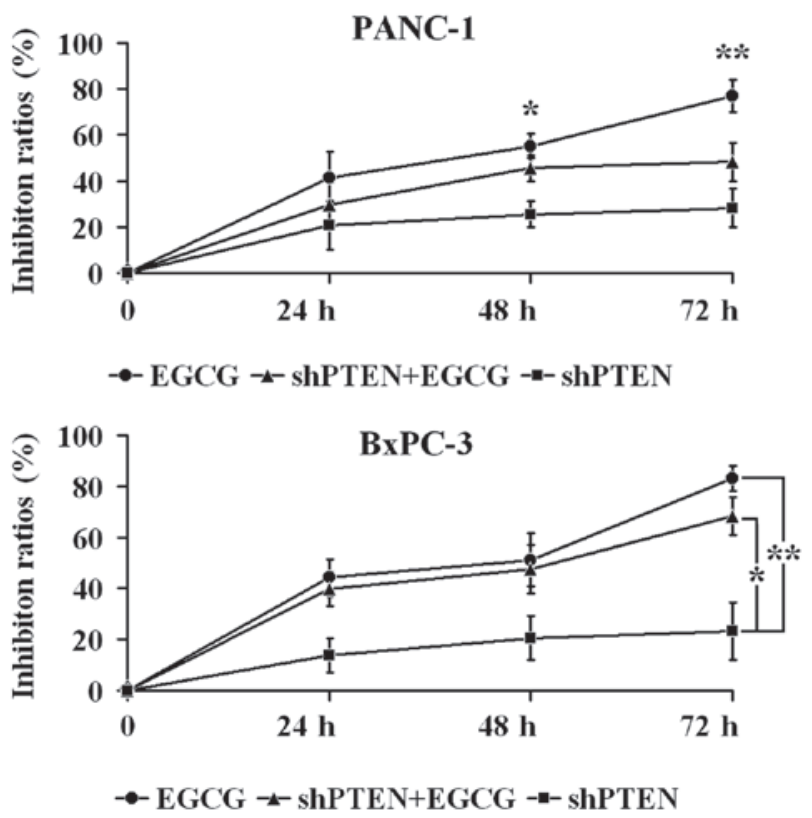

Figure 2. Effect of EGCG on prostate cancer cell proliferation via PTEN. PANC-1 and BxPC-3 cells with or without PTEN knockdown were cultured in medium with or without $40 \mu \mathrm{g} / \mathrm{ml}$ EGCG. Cells were harvested at 24 , 48 and $72 \mathrm{~h}$, and the proliferative potential was assessed. Data presented are the mean of three independent experiments. ${ }^{*} \mathrm{P}<0.05,{ }^{* *} \mathrm{P}<0.01$ vs. shPTEN group. EGCG, (-)-epigallocatechin-3-gallate; PTEN, phosphatase and tensin homolog deleted on chromosome 10; sh, short hairpin.

group was not significantly different compared with shPTEN group $(\mathrm{P}>0.05)$. In BxPC-3 cells, the inhibition ratios in the EGCG group were significantly greater compared with the shPTEN group $(\mathrm{P}<0.01)$. Additionally, the inhibition ratios in the shPTEN+EGCG group was significantly higher than the shPTEN group $(\mathrm{P}<0.05)$.

Effect of EGCG on PC cell apoptosis via PTEN. PANC-1 and BxPC-3 cells with or without PTEN knockdown were cultured in medium with or without $40 \mu \mathrm{g} / \mathrm{ml} \mathrm{EGCG} \mathrm{for}$ 
PANC-1
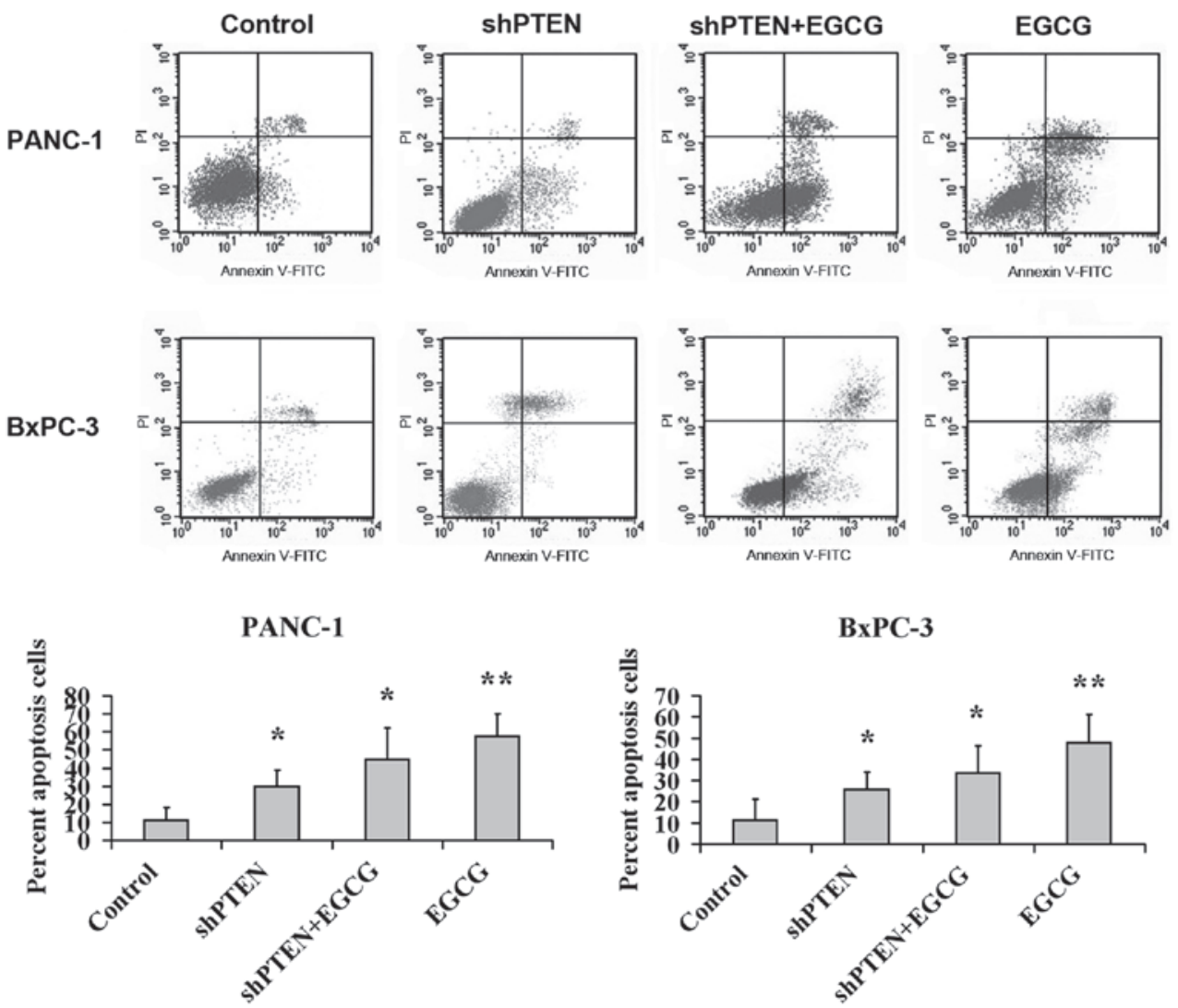

Figure 3. Effect of EGCG on apoptosis of prostate cancer cells via PTEN. PANC-1 and BxPC-3 cells with or without PTEN knockdown were treated with $40 \mu \mathrm{g} / \mathrm{ml}$ EGCG for $48 \mathrm{~h}$. The apoptotic rate of cells was analyzed by flow cytometry. Cells in the lower right quadrant were considered to be in early apoptosis, and cells in the upper right quadrant in late apoptosis. ${ }^{*} \mathrm{P}<0.05,{ }^{* *} \mathrm{P}<0.01 \mathrm{vs}$. control group. EGCG, (-)-epigallocatechin-3-gallate; PTEN, phosphatase and tensin homolog deleted on chromosome 10; sh, short hairpin.

$48 \mathrm{~h}$, and the apoptotic rate was analyzed by flow cytometry (Fig. 3). This indicated that the apoptotic ratios in the EGCG group were substantially higher compared with the control (untreated) group $(\mathrm{P}<0.01)$. Additionally, the apoptotic rates in the shPTEN and shPTEN+EGCG groups were substantially higher than the control group $(\mathrm{P}<0.05)$.

EGCG regulates the expression of genes and proteins in the PI3K/Akt/mTOR pathway in PC cells via PTEN. PANC-1 and BxPC-3 cells with or without PTEN knockdown were cultured in medium with or without $40 \mu \mathrm{g} / \mathrm{ml}$ EGCG for $48 \mathrm{~h}$, and the mRNA expression of PI3K, PTEN, Akt and mTOR is presented in Fig. 4. The mRNA expression levels of PTEN in the shPTEN group were significantly lower compared with the control (untreated) group $(\mathrm{P}<0.05)$. Furthermore, the mRNA expression of PTEN in the EGCG group was significantly higher compared with the control group $(\mathrm{P}<0.01)$. However, the mRNA expression levels of PTEN in the shPTEN+EGCG group did not differ compared with the control group $(\mathrm{P}>0.05)$.

Subsequently, the effect of EGCG on the protein expression of PI3K, PTEN, Akt, p-Akt, mTOR and p-mTOR in PANC-1 and BxPC-3 cells was investigated with or without PTEN knockdown (Fig. 4). The $\beta$-actin expression levels in the treated groups were unaltered compared with the control groups $(\mathrm{P}>0.05)$. The protein expression levels of PTEN in the shPTEN group were significantly lower compared with the control (untreated) group $(\mathrm{P}<0.01)$. Furthermore, the expression of PTEN in the EGCG group was markedly higher compared with the control group $(\mathrm{P}<0.01)$. However, the expression of PTEN in the shPTEN+EGCG group did not differ compared with the control group $(\mathrm{P}>0.05)$. The expression levels of p-Akt and p-mTOR in the shPTEN group were significantly greater compared with the control (untreated) group $(\mathrm{P}<0.05)$. Additionally, the p-Akt and p-mTOR expression levels in the EGCG groups were significantly lower compared with the control group $(\mathrm{P}<0.05)$. However, the $\mathrm{p}-\mathrm{Akt}$ and $\mathrm{p}$-mTOR expression levels in the shPTEN+EGCG group were unaltered compared with the control group $(\mathrm{P}>0.05)$.

\section{Discussion}

In the present study, using PANC-1 cells the inhibition ratio in normal cells following EGCG treatment at 48 and $72 \mathrm{~h}$ was observed to be significantly higher compared with shPTEN cells $(\mathrm{P}<0.05$ and $\mathrm{P}<0.01$, respectively), with the inhibition ratio in shPTEN cells following EGCG treatment unaltered compared with shPTEN cells ( $\mathrm{P}>0.05)$. In BxPC-3 cells, the inhibition ratios in normal cells following EGCG treatment were significantly higher compared with shPTEN cells $(\mathrm{P}<0.01)$, with the inhibition ratios in shPTEN cells following EGCG treatment substantially higher than in the shPTEN cells $(\mathrm{P}<0.05)$. These data indicate that PTEN was involved in EGCG inhibiting PC cell proliferation, with the knockdown 
A

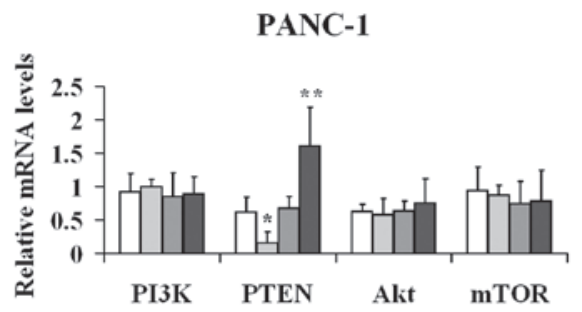

$\square$ Control $\square$ shPTEN $\square \operatorname{shPTEN+EGCG~} \square$ EGCG

$\mathbf{B}$
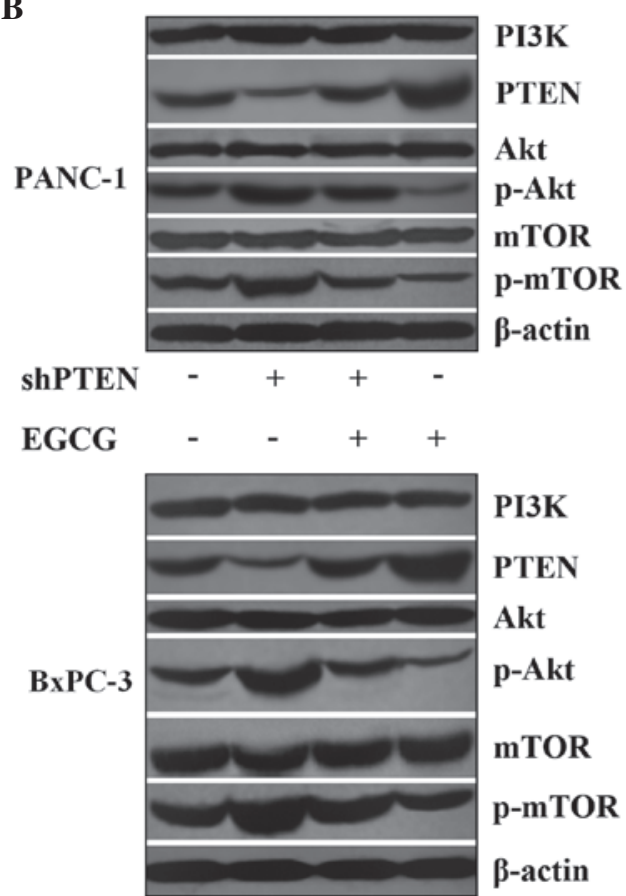

BxPC-3

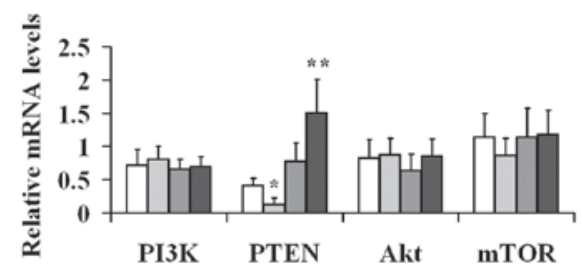

$\square$ Control $\square \operatorname{shPTEN} \square \operatorname{shPTEN+EGCG} \square$ EGCG

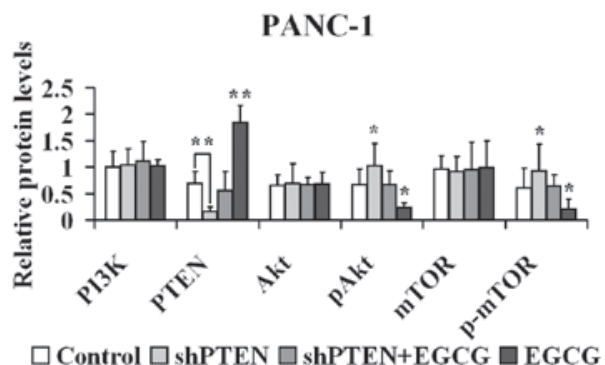

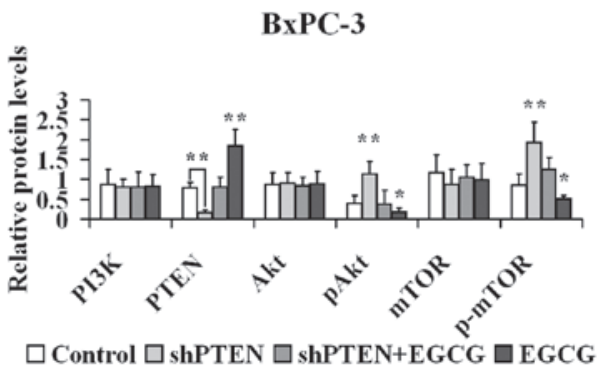

Figure 4. Effect of EGCG on expression of genes and proteins involved in the PI3K/Akt/mTOR pathway in prostate cancer cells via PTEN. (A) PANC-1 and BxPC-3 cells with or without PTEN knockdown were treated with $40 \mu \mathrm{g} / \mathrm{ml}$ EGCG for $48 \mathrm{~h}$. PI3K, PTEN, Akt and mTOR mRNA expression levels were measured by reverse transcription-polymerase chain reaction. (B) PANC-1 and BxPC-3 cells with or without PTEN knockdown were treated with $40 \mu \mathrm{g} / \mathrm{ml}$ EGCG for 48 h. PI3K, PTEN, Akt, pAkt, mTOR and p-mTOR expression levels were measured by western blotting. "P<0.05, ${ }^{* *}$ P $<0.01$ vs. control group. EGCG, (-)-epigallocatechin-3-gallate; PI3K, phosphoinositide 3-kinase; Akt, protein kinase B; mTOR, mechanistic target of rapamycin; PTEN, phosphatase and tensin homolog deleted on chromosome 10; sh, short hairpin; p-, phosphorylated.

of PTEN reducing the inhibitory effect of EGCG on PC cell proliferation. Furthermore, the previous findings support the present study. In a previous study, the proliferation of PANC-1 cells was inhibited following treatment with $40 \mu \mathrm{g} / \mathrm{ml} \mathrm{EGCG}$ for 24, 48 and $72 \mathrm{~h}$ (22). In addition, Zhang et al (25) reported that loss of PTEN promoted proliferation and invasion in PC cells, and Ma et al (26) demonstrated that knockdown of PTEN was able to upregulate cell invasiveness and proliferation in PC cells. Furthermore, Lyn-Cook et al (27) demonstrated that EGCG suppressed pancreatic cell growth by approximately 90\%. Differences in the methods or cell lines used in these studies may explain the discrepancies between these studies and the present study.

The current study indicated that the apoptotic rates in normal cells following EGCG treatment were significantly higher compared with the control group $(\mathrm{P}<0.01)$, and the apoptotic rates in the shPTEN cells with or without EGCG treatment were significantly higher compared with the control group $(\mathrm{P}<0.05)$. These results suggested that PTEN was involved in EGCG promoting PC cell apoptosis, and that the absence of PTEN may attenuate the apoptosis-promoting ability of EGCG in PC cells. These results are supported by previous studies. In a previous study, the apoptosis ratio in PANC-1 cells following $40 \mu \mathrm{g} / \mathrm{ml}$ EGCG treatment over $24 \mathrm{~h}$ was $28.56 \pm 1.56 \%$ (22). Qanungo et al (28) reported that EGCG induced the apoptosis of human PC Mia Paca-2 cells and that the apoptotic rate was 2.5-25\% following treatment with $0.025-0.2 \mathrm{mM}$ EGCG for $24 \mathrm{~h}$. The differences in the cell types and EGCG concentrations used in these previous studies may account for the variation in these rates.

PTEN is an important negative modulator of the $\mathrm{PI} 3 \mathrm{~K} / \mathrm{Akt} / \mathrm{mTOR}$ pathway, as it can weaken upstream signals. Deactivation of PTEN leads to activated PI3K/Akt/mTOR signaling. In the present study, the mRNA and protein expression of PTEN in normal cells following treatment with EGCG were significantly higher compared with the controls $(\mathrm{P}<0.01)$. The mRNA and protein expression levels of PTEN in shPTEN cells following EGCG treatment were comparable with the control cells $(\mathrm{P}>0.05)$. The expression levels of $\mathrm{p}$-Akt and p-mTOR in shPTEN cells were significantly higher compared with the controls $(\mathrm{P}<0.05)$, whilst the p-Akt and $\mathrm{p}$-mTOR expression levels in normal cells treated with EGCG alone were 
significantly lower compared with the control cells $(\mathrm{P}<0.05)$. The expression levels of p-Akt and p-mTOR in shPTEN cells treated with EGCG were comparable with the control cells $(\mathrm{P}>0.05)$. Previous studies have demonstrated that EGCG is able suppress the PI3K/Akt/mTOR pathway by downregulating p-Akt and p-mTOR expression based on the presence of PTEN, instead of regulating Akt and mTOR $(22,29)$. These data indicate that EGCG-induced upregulation of PTEN expression is a prohibitive mechanism on the PI3K/Akt/mTOR pathway and that the loss of PTEN may attenuate the inhibitory effect of EGCG on the PI3K/Akt/mTOR pathway in human PC cells. However, previous studies support the results of the present study. In a previous study, EGCG upregulated the expression levels of PTEN and downregulated the expressions of p-Akt and p-mTOR in PANC-1 cells (22). Additionally, Zhang et al (25) reported that loss of PTEN resulted in increased expression of p-Akt and p-mTOR in PC cells and Shankar et al (30) reported that EGCG inhibited the phosphorylation of PI3K and p-Akt in PC tissues and promoted PTEN expression, however with no influence on Akt. Nevertheless, certain studies have indicated that EGCG is able to modulate the expression levels of PI3K, mTOR or Akt in certain types of cancer. Shen et al (31) reported that EGCG treatment resulted in a reduction in the mRNA and protein expression levels of PI3K and Akt in hepatoma, and $\mathrm{Li}$ et al (32) found that loss of PTEN resulted in increased expression levels of Akt, p-Akt, and p-mTOR in endometrial cancer cells. Additionally, Van Aller et al (33) indicated that EGCG was able to inhibit the expression of PI3K, mTOR and p-Akt in MDA-MB-231 and A549 cells, and Shimizu et al (34) demonstrated that EGCG can inhibit the expression levels of Akt and p-Akt in colorectal cancer xenograft tumors. Furthermore, Shirakami et al (35) reported that EGCG can repress Akt expression in human hepatoma $\mathrm{HuH} 7$ cell xenografts, and Ichimatsu et al (36) reported that EGCG can repress the activation of PI3K in JB6Cl41 cells. However, alterations in the expression levels of PI3K, Akt and mTOR were not observed in the current study, therefore further study is required.

In conclusion, EGCG was able to inhibit proliferation and induce apoptosis in PC cells via PTEN, with the loss of PTEN reducing the ability of EGCG to inhibit proliferation and promote apoptosis in PC cells. In addition, EGCG is able to downregulate the expression levels of p-Akt and p-mTOR to regulate then PI3K/Akt/mTOR pathway via PTEN. Furthermore, this regulatory effect may contribute to the apoptosis-inducing and anti-proliferative properties of EGCG. However, further study is required to fully elucidate the regulatory effect of EGCG on components downstream of the PI3K/Akt/mTOR signal pathway.

\section{Acknowledgements}

The current study was supported by the Science and Technology Research Grant of Education Department of Heilongjiang Province, China (grant no. 12541921).

\section{References}

1. Ghaneh P, Costello E and Neoptolemos JP: Biology and management of pancreatic cancer. Gut 56: 1134-1152, 2007.
2. Zhang Y and Shi X: Progress in the chemotherapy of pancreatic carcinoma. World Chin J Digestology 17: 1422-1426, 2009.

3. Suganuma M, Okabe S, Sueoka N, Sueoka E, Matsuyama S, Imai K, Nakachi K and Fujiki H: Green tea and cancer chemoprevention. Mutat Res 428: 339-344, 1999.

4. Shankar S, Ganapathy S and Srivastava RK: Green tea polyphenols: Biology and therapeutic implications in cancer. Front Biosci 12: 4881-4899, 2007.

5. Yang CS, Ju J, Lu G, Xiao H, Hao X, Sang S and Lambert JD: Cancer prevention by tea and tea polyphenols. Asia Pac J Clin Nutr 17 (Suppl 1): S245-S248, 2008.

6. Hastak K, Gupta S, Ahmad N, Agarwal MK, Agarwal ML and Mukhtar H: Role of P53 and NF-kappaB inepigallocatechin3-gallate-induced apoptosis of LNCaP cells. Oncogene 22: 4851-4859, 2003.

7. Maeda-Yamamoto M, Suzuki N, Sawai Y, Miyase T, Sano M, Hashimoto-Ohta A and Isemura M: Association of suppression of extracellular signal-regulated kinase phosphorylation by epigallocatechin gallate with the reduction of matrix metalloproteinase activities in human fibrosarcoma HT1080 cells. J Agic Food Chem 51: 1858-1863, 2003.

8. Roy M, Chakrabarty S, Sinha D, Bhattacharya RK and Siddiqi M: Anticlastogenic, antigenotoxic and apoptoic antivity of epigallocatechin gallate: A green tea polyphenol. Mutat Res 523-524: 33-41, 2003.

9. Mittal A, Pate MS, Wylie RC, Tollefsbol TO and Katiyar SK: EGCG down-regulates telomerase in human breast carcinoma MCF-7 cells, leading to suppression of cell viability and induction of apotosis. Int J Oncol 24: 703-710, 2004.

10. Mukhtar H and Ahmad N: Tea polyphenols: Prevention of cancer and optimizing health. Am J Clin Nutr 71 (Suppl 6): S1698-S1702; discussion S1703-S1704, 2000.

11. Lambert JD and Yang CS: Cancer chemopreventive activity and bioavailability of tea and tea polyphenols. Mutat Res 523-524: 201-208, 2003.

12. Bode AM and Dong Z: Targeting signal transduction pathways by chemopreventive agents, Mutat Res 555: 33-51, 2004.

13. Yang CS, Chung JY, Yang G, Chhabra SK and Lee MJ: Tea and tea polyphenols in cancer prevention. J Nutr 130 (2S Suppl): S472-S478, 2000.

14. Leone M, Zhai D, Sareth S, Kitada S, Reed JC and Pellecchia M: Cancer prevention by tea polyphenols is linked to their direct inhibition of antiapoptotic Bcl-2-family proteins. Cancer Res 63 : 8118-8121, 2003

15. Pellecchia M and Reed JC: Inhibition of anti-apoptotic Bcl-2 family proteins by natural polyphenols: New avenues for cancer chemoprevention and chemotherapy. Curr Pharm Des 10: 1387-1398, 2004.

16. Yang CS, Lambert JD, Hou Z, Ju J, Lu G and Hao X: Molecular targets for the cancer preventive activity of tea polyphenols. Mol Carcinog 45: 431-435, 2006.

17. Surh YJ: Cancer chemoprevention with dietary phytochemicals. Nat Rev Cancer 3: 768-780, 2003.

18. Kanwar J, Taskeen M, Mohammad I, Huo C, Chan TH and Dou QP: Recent advances on tea polyphenols. Front Biosci (Elite Ed) 4: 111-131, 2012.

19. Morgan TM, Koreckij TD and Corey E: Targeted therapy for advanced prostate cancer: Inhibition of the PI3K/Akt/mTOR pathway. Curr Cancer Drug Targets 9: 237-249, 2009.

20. Downward J: PI3-kinase, Akt and cell survival. Semin Cell Dev Biol 15: 177-182, 2004.

21. Xu G, Zhang W, Bertram P, Zheng XF and McLeod H: Pharmacogenomic profiling of the PI3K/PTEN-AKT-mTOR pathway in common human tumors. Int J Oncol 24: 893-900, 2004.

22. Liu S, Wang XJ, Liu Y and Cui YF: PI3K/AKT/mTOR signaling is involved in (-)-epigallocatechin-3-gallate-induced apoptosis of human pancreatic carcinoma cells. Am J Chin Med 41: 629-642, 2013.

23. Lin CF, Young KC, Bai CH, Yu BC, Ma CT, Chien YC, Chiang CL, Liao CS, Lai HW and Tsao CW: Rosiglitazone regulates anti-inflammation and growth inhibition via PTEN Biomed Res Int 2014: 787924: 2014.

24. Xie F, Su M, Qiu W, Zhang M, Guo Z, Su B, Liu J, Li X and Zhou L: Kaempferol promotes apoptosis in human bladder cancer cells by inducing the tumor suppressor, PTEN. Int J Mol Sci 14: 21215-21226, 2013.

25. Zhang Y, Zhang J, Xu K, Xiao Z, Sun J, Xu J, Wang J and Tang Q: PTEN/PI3K/mTOR/B7-H1 signaling pathway regulates cell progression and immuno-resistance in pancreatic cancer. Hepatogastroenterology 60: 1766-1772, 2013. 
26. Ma J, Sawai H, Matsuo Y, Ochi N, Yasuda A, Takahashi H, Wakasugi T, Funahashi H, Sato M and Takeyama H: IGF-1 mediates PTEN suppression and enhances cell invasion and proliferation via activation of the IGF-1/PI3K/Akt signaling pathway in pancreatic cancer cells. J Surg Res 160: 90-101, 2010.

27. Lyn-Cook BD, Rogers T, Yan Y, Blann EB, Kadlubar FF and Hammons GJ: Chemopreventive effects of tea extracts and various components on human pancreatic and prostate tumor cells in vitro. Nutr Cancer 35: 80-86, 1999.

28. Qanungo S, Das M, Haldar S and Basu A: Epigallocatechin-3-gallate induces mitochondrial membrane depolarization and caspase-dependent apoptosis in pancreatic cancer cells. Carcinogenesis 26: 958-967, 2005.

29. Wang LY, Li X and Han YZ: Neuroprotection by epigallocatechin gallate against bupivacaine anesthesia induced toxicity involves modulation of PI3/Akt/PTEN signalling in N2a and SH-SY5Y cells. Int J Clin Exp Med 8: 15065-15075, 2015.

30. Shankar S, Marsh L and Srivastava RK: EGCG inhibits growth of human pancreatic tumors orthotopically implanted in Balb $\mathrm{C}$ nude mice through modulation of FKHRL1/FOXO3a and neuropilin. Mol Cell Biochem 372: 83-94, 2013.

31. Shen X, Zhang Y, Feng Y, Zhang L, Li J, Xie YA and Luo X: Epigallocatechin-3-gallate inhibits cell growth, induces apoptosis and causes $\mathrm{S}$ phase arrest in hepatocellular carcinoma by suppressing the AKT pathway. Int J Oncol 44: 791-796, 2014.
32. Li T, Yang Y, Li X, Xu C and Meng L: EGFR- and AKT-mediated reduction in PTEN expression contributes to tyrphostin resistance and is reversed by mTOR inhibition in endometrial cancer cells. Mol Cell Biochem 361: 19-29, 2012.

33. Van Aller GS, Carson JD, Tang W, Peng H, Zhao L, Copeland RA, Tummino PJ and Luo L: Epigallocatechin gallate (EGCG), a major component of green tea, is a dual phosphoinositide-3-kinase/mTOR inhibitor. Biochem Biophys Res Commun 406: 194-199, 2011.

34. Shimizu M, Shirakami Y, Sakai H, Yasuda Y, Kubota M, Adachi S, Tsurumi H, Hara Y and Moriwaki H: (-)-Epigallocatechin gallate inhibits growth and activation of the VEGF/VEGFR axis in human colorectal cancer cells. Chem Biol Interact 185: 247-252, 2010.

35. Shirakami Y, Shimizu M, Adachi S, Sakai H, Nakagawa T, Yasuda Y, Tsurumi H, Hara $Y$ and Moriwaki $H$ : (-)-Epigallocatechin gallate suppresses the growth of human hepatocellular carcinoma cells by inhibiting activation of the vascular endothelial growth factor-vascular endothelial growth factor receptor axis. Cancer Sci 100: 1957-1962, 2009.

36. Ichimatsu D, Nomura M, Nakamura S, Moritani S, Yokogawa K, Kobayashi S, Nishioka T and Miyamoto K: Structure-activity relationship of flavonoids for inhibition of epidermal growth factor-induced transformation of JB6 Cl 41 cells. Mol Carcinog 46: 436-445, 2007. 